\title{
Atualização em Sepse: Ressuscitação Hemodinâmica
}

\section{A ressuscitação hemodinâmica precoce guiada por metas está indicada em todos os pacientes com sepse grave?}

a) Está recomendada para pacientes sépticos graves que apresentem hipotensão refratária a volume e/ou lactato sérico elevado ( $\geq 4 \mathrm{mmol} / \mathrm{l})$

b) Deve ser utilizada na ausência de hipotensão;

c) A meta é pressão venosa central (PVC) acima de $12 \mathrm{mmHg}$;

d) Deve ser utilizada na ausência de hiperlactatemia.

\section{Existe um vasopressor ideal para ser utilizado no paciente séptico?}
a) A dopamina é mais potente que a noradrenalina;
b) A associação de vasopressina com noradrenalina não traz benefícios sobre a mortalidade;
c) A adrenalina é o fármaco de primeira escolha nesses pacientes;
d) A vasopressina é fármaco de primeira escolha.

3. Existe um inotrópico ideal para ser utilizado no paciente séptico com sinais de disfunção miocárdica?
a) O débito cardíaco deve ser supra-normalizado;
b) A terapia com dobutamina está indicada independente da SvcO2;
c) A dobutamina é o inotrópico de escolha;
d) Na hipotensão o uso de vasopressor associado a dobutamina está contra-indicado.

4. Existe benefício em monitorar a saturação venosa central de oxigênio (svco2)?
a) A restauração da estabilidade hemodinâmica baseada na PAM resulta em benefício em termos de prognóstico;
b) A estabilidade hemodinâmica baseada em PVC é suficiente para a restauração da oxigenação tecidual;
c) A terapêutica baseada na SvcO2 não reduz a mortalidade;
d) A terapêutica baseada na SvcO2 e na sua normalização precoce resulta na recuperação do fluxo sanguíneo;

\section{Há vantagens prognósticas em monitorizar a svco2 após a fase de ressuscitação?}

a) A meta deve ser cumprida nas primeiras 24 horas de tratamento;

b) Atingir precocemente a meta terapêutica de $\mathrm{SvcO} 2>70 \%$ não tem impacto sobre a mortalidade;

c) A meta deve ser cumprida o mais precocemente possível, preferencialmente nas 6 primeiras horas de tratamento;

d) Atingir a SvcO2 > 70\% não altera o tempo de ventilação mecânica.

\section{Respostas ao Cenário Clínico: Tabagismo (Parte 3) [Publicado na RAMB 2010; 56(4)]}

1. A terapia de reposição de nicotina pode levar a parto prematuro (Alternativa $\mathrm{C}$ ).

2. A abordagem para a cessação do tabagismo deve ser recomendada para os adolescentes é o aconselhamento (Alternativa B).

3. Especial atenção durante o tratamento com vareniclina o qual pode estar associado a humor depressivo, agitação e ideação ou comportamento suicida (Alternativa C)

4. Documentar a situação do paciente: fumante, nunca fumante, ex-fumante, está entre os itens para intervenção hospitalar (Alternativa $\mathrm{C}$ ).

5. A presença de depressão é obstáculo para a cessação em mulheres (Alternativa B). 